\title{
Supersymmetric Harry Dym Type Equations
}

\author{
Q. P. Liu \\ CCAST(World Laboratory), \\ P.O.Box 8730, Beijing 100080, China \\ and \\ Department of Mathematics, \\ Beijing Graduate School(\# 65), \\ China University of Mining and Technology, \\ Beijing 100083, China円
}

\begin{abstract}
A supersymmetric version is proposed for the well known Harry Dym system. A general class super Lax operator which leads to consistent equations is considered.
\end{abstract}

${ }^{1}$ mailing address 
During last ten years or so, super extensions of integrable models have been a subject arrested much attention. The consequence of such study is that a number of the well known integrable systems are embedded in the context of super systems. In particular, we mention here the super Sine-Gordon[1], Korteweg-de $\operatorname{Vries}(\mathrm{KdV})[2,3,4]$ and nonlinear Schrodinger equations[5] and super Kadomtsev-Petviashvili hierarchy[2], etc..(see [6] for more references).

We note that two type super extensions for a given integrable system may exist, that is, supersymmetric and fermionic extensions. In KdV case, this corresponds Manin-Radul' version[2] and Kupershmidt's version[4] respectively. Apart from KdV system, Harry Dym(HD) equation is also well known. Very recently, it is found that HD equation is not just a mathematically interested model and it possesses physical applications[7]. Thus, it is interesting to construct a super analogy of HD equation. In this regard, a fermionic HD model is known from Kupershmidt's work[8] while a generic supersymmetric HD (sHD) system is still lacking to the best of my knowledge. The aim of this Note is to propose such a model.

For convenience, we fix our notations at the very beginning: denoting even variables by Latin letters and odd variables by Greek letters; index $\geq r$ of a operator always means the projection to the part of order greater than $D^{r}$ (including the term $D^{r}$ itself).

Let us first recall some basic facts of HD equation. The equation reads

$$
w_{t}=\frac{1}{4} w^{3} w_{x x x}
$$

or

$$
u_{t}=\frac{1}{4}\left(u^{\frac{3}{2}} u_{x x x}-\frac{3}{2} u^{\frac{1}{2}} u_{x} u_{x x}+\frac{3}{4} u^{-\frac{1}{2}} u_{x}^{3}\right)
$$

The link between them is $u=w^{2}$.

It is known that HD equation(2) has the following Lax operator

$$
L_{H D}=u \partial^{2}
$$

and the Lax equation is

$$
L_{H D_{t}}=\left[P, L_{H D}\right]
$$

where $P=\left(L^{\frac{3}{2}}\right)_{\geq 4}$.

Our candidate for the supersymmetric Harry Dym(sHD) equation is:

$$
\begin{aligned}
u_{t}= & \left(\frac{1}{4} u^{\frac{3}{2}} u_{x x x}-\frac{3}{8} u^{\frac{1}{2}} u_{x} u_{x x}+\frac{3}{16} u^{-\frac{1}{2}} u_{x}^{3}\right)+\frac{3}{8} u^{\frac{1}{2}} \alpha\left(D u_{x x}\right)-\frac{3}{4} u^{\frac{1}{2}} \alpha \alpha_{x x}- \\
& \frac{3}{32} u^{-\frac{3}{2}} u_{x}^{2} \alpha(D u)+\frac{3}{16} u^{-\frac{1}{2}} u_{x x} \alpha(D u)+\frac{3}{8} u^{-\frac{1}{2}} u_{x} \alpha_{x}(D u)-\frac{3}{8} u^{-\frac{1}{2}} u_{x} \alpha\left(D u_{x}\right)- \\
& \frac{3}{8} u^{\frac{1}{2}} \alpha_{x x}(D u)+\frac{3}{16} u^{-\frac{3}{2}} u_{x} \alpha(D \alpha)(D u)-\frac{3}{8} u^{-\frac{1}{2}} \alpha\left(D \alpha_{x}\right)(D u)+\frac{3}{4} u^{-\frac{1}{2}} \alpha \alpha_{x} u_{x} . \\
\alpha_{t}= & \frac{1}{4} u^{\frac{3}{2}} \alpha_{x x x}+\frac{3}{8} u^{\frac{1}{2}} \alpha\left(D \alpha_{x x}\right)+\frac{3}{16} u^{-\frac{1}{2}} u_{x}^{2} \alpha_{x}-\frac{3}{8} u^{\frac{1}{2}} u_{x x} \alpha_{x}-\frac{9}{32} u^{-\frac{3}{2}} u_{x}^{2} \alpha(D \alpha)+ \\
& \frac{3}{16} u^{-\frac{1}{2}} u_{x x} \alpha(D \alpha)+\frac{3}{8} u^{-\frac{1}{2}} u_{x} \alpha_{x}(D \alpha)-\frac{3}{8} u^{\frac{1}{2}} \alpha_{x x}(D \alpha)+\frac{3}{16} u^{-\frac{3}{2}} u_{x} \alpha(D u) \alpha_{x}- \\
& \frac{3}{8} u^{-\frac{1}{2}} \alpha\left(D u_{x}\right) \alpha_{x}+\frac{3}{16} u^{-\frac{3}{2}} u_{x} \alpha(D \alpha)^{2}+\frac{3}{16} u^{-\frac{3}{2}} \alpha(D u) \alpha_{x}(D \alpha)-\frac{3}{8} u^{-\frac{1}{2}} \alpha(D \alpha)\left(D \alpha_{x}\right)
\end{aligned}
$$

Where $D=\theta \partial+\partial_{\theta}, \mathrm{u}$ is a super even variable and $\alpha$ is a super odd variable. Since the system is formulated in superderivartive and super fields, the supersymmetry is manifest. If we set the odd field variable $\alpha$ to zero, we get the HD equation(2), thus the system (5) deserves the name of sHD. 
The system(5) has the following Lax representation:

$$
L_{t}=[P, L]
$$

where $L=u \partial^{2}+\alpha \partial D$, and $P=\left(L^{\frac{3}{2}}\right)_{\geq 3}=u^{\frac{3}{2}} \partial^{3}+\frac{3}{2} u^{\frac{1}{2}} \alpha \partial^{2} D+\left(\frac{3}{4} u^{\frac{1}{2}} u_{x}+\frac{3}{8} u^{-\frac{1}{2}} \alpha(D u)\right) \partial^{2}+\left(\frac{3}{4} u^{\frac{1}{2}} \alpha_{x}+\right.$ $\left.\frac{3}{8} u^{-\frac{1}{2}} \alpha(D \alpha)\right) \partial D$..

Noticing the sHD system can be reformulated in Lax form, we see that this kind of representation is nonstandard in Kupershmidt sense[9](see also Kiso[10]). A detailed presentation of nonstandard Lax representation can be found in[11]. This remark suggests us to consider more general operator:

$$
L=u \partial^{2}+\alpha \partial D+v \partial+\beta D+w
$$

Taking L as a Lax operator, we may construct integrable systems by means of fractional power method. It is not difficult to verify that the following four cases occur:

Case 1:

$$
L_{t}=\left[\left(L^{\frac{3}{2}}\right)_{\geq 0}, L\right]
$$

The standard argument shows that the system is consistent: since $\left[\left(L^{\frac{3}{2}}\right)_{\geq 0}, L\right]=-\left[\left(L^{\frac{3}{2}}\right)_{<0}, L\right]$, the right hand side of (8) is a diffenertial operator of form $A \partial+\gamma D+B$. Thus, we may set $u=1, \alpha=0$. However, this implies $v=0$ and we end with Manin-Radul case[2,3].

Case 2:

$$
L_{t}=\left[\left(L^{\frac{3}{2}}\right)_{\geq 1}, L\right]
$$

The same argument leads to the conclusion: we may set $u=1, \alpha=0$. Thus, we here have a system of three equations. It is easy to see that we may further set $w=0$. This last case was noticed by Inami and Kanno[6].

Case 3:

$$
L_{t}=\left[\left(L^{\frac{3}{2}}\right)_{\geq 2}, L\right]
$$

The general case will lead to a system involved five fields. However, we may have a subsystem which only have three fields.

Case 4:

$$
L_{t}=\left[\left(L^{\frac{3}{2}}\right)_{\geq 3}, L\right]
$$

As above, this system involved all five fields. A reduction gives us the sHD system(5).

Remarks.

(1). In the pure bosonic case, we only have three cases which corresponds to KdV, MKdV and Harry-Dym systems respectively[10,11].

(2). A simple calculation shows that the next one $L_{t}=\left[\left(L^{\frac{3}{2}}\right)_{\geq 4}, L\right]$ will not lead to any consistent system. Thus, we here have only four cases.

For a general even order diffenertial operator

$$
L=\sum_{i=0}^{n} u_{i} D^{2 i}+\sum_{i=1}^{n} \alpha_{i} D^{2 i-1}
$$

we may consider the following Lax equation

$$
L_{t}=\left[\left(L^{\frac{k}{n}}\right)_{\geq r}, L\right], \quad r=0,1,2,3
$$


The $r=0,1$ cases are considered in [12] and [6] respectively. It is pointed out that one may set the fields $u_{n}=\alpha_{n}=u_{n-1}=0$ to zero in the case $r=0$ and $u_{n}=\alpha_{n}=u_{0}=0$ in the case $r=1$. When $r=2$ and $r=3$, all the field variables can be taken as dynamical variables. However, the following reductions or restrictions are feasible: $u_{0}=0, \alpha_{1}=0$ for $r=2$ case and $u_{1}=u_{0}=0, \alpha_{1}=0$ for the case $r=3$.

The systems (13) are integrable in the sense that they consist of commuting flows. The proof of this statement is not difficult: the first two cases are proved in the cited references. The proof for last two cases follows from the standard argument.

We conclude this Note with the remarks in order:

(1). We see that for the operator(12), we have four cases mentioned above. This phenomenon is based on the following algebraic decompositions:

$$
g=\left\{\sum_{i} u_{i} D^{i}\right\}=g_{\geq r} \oplus g_{<r}, \quad r=0,1,2,3 .
$$

(2). It would be interesting to study the Hamiltonian structures of our system sHD. We know that HD equation is not only Hamiltonian but bi-Hamiltonian. We suspect that it is also the case for the sHD.

(3). It is proved that the case $r=0$ and case $r=1$ are gauge related each other[6]. It is important to study the relationship between $r=1$ case and the $r=2$ case and $r=3$ case.

(4). We may construct the flows in terms of Sato's approach. The candidate of pseudodiffenertial operator is $L=u_{0} \partial+\alpha_{0} D+\ldots$

(5). In a recent paper[14], Darboux transformations for sKdV are constructed. The same consideration will be interesting for general cases.

\section{Acknowledgement}

I should like to thank Boris Kupershmidt for communications on sHD. It is my pleasure to thank the referee for the useful comments. This work is supported by National Natural Science Foundation of China.

\section{References}

[1] Chainchian M and Kulish P P 1978 Phys.Lett. 78B 413.

[2] Manin Yu and Rudal A 1985 Commun. Math. Phys. 9865.

[3] Mathieu P 1988 J. Math. Phys. 282499.

[4] Kupershmidt B A 1984 Phys. Lett. 102A 213.

[5] Roelofs G H M and Kersten P H M 1992 J. Math. Phys. 332185.

[6] Inami T and Kanno H 1992 Inter. J. Mod. Phys. 7 (Suppl 1A) 419; Morois C and Pizzocchero L 1994 J. Math. Phys. 352397.

[7] Kadanoff L P 1990 Phys. Rev. Lett. 652986.

[8] Kupershmidt B A 1987 Elements of Superintegrable Systems, D. Reidel, Dordrecht.

[9] Kupershmidt B A 1985 Commun. Math. Phys. 9951.

[10] Kiso K 1990 Progr. Theor. Phys. 831108.

[11] Konopelchenko B G and Oevel W 1993 Publ. RIMS, Kyoto University 291.

[12] Figueroa-O'Farill J Ramos E and Mas J 1991 Rev. Math. Phys. 3 479; Oevel W and Popowicz Z 1991 Commun. Math. Phys. 139441.

[13] Oevel W and Rogers C 1993 Rev. Math. Phys. 5299.

[14] Liu Q P 1994 Darboux Transformation for Supersymmetric Korteweg-de Vries Equations, preprint, ASITP-94-41, Lett. Math. Phys. (in press). 